Internist 2010 $51: 5-5$

DOI 10.1007/s00108-009-2562-0

Online publiziert: 16. Januar 2010

๑) Springer-Verlag 2010

\author{
H. Haller ${ }^{1}$-W.L. Gross ${ }^{2}$ \\ ${ }^{1}$ Abteilung Nephrologie, Medizinische Hochschule Hannover \\ 2 Poliklinik für Rheumatologie, Universitätsklinikum Schleswig- \\ Holstein, Lübeck und Klinikum Bad Bramstedt
}

\title{
Fibrosierende Erkrankungen
}

Die vorliegende Ausgabe von „Der Internist" beschäftigt sich mit dem Thema „Fibrosierende Erkrankungen“. Die Fibrose und Sklerosierung von Gewebe stellt häufig die Endstrecke im Verlauf von chronischen Erkrankungen dar. Die massive Bildung von Bindegewebe mit zunehmendem Verlust der Organfunktion sind die typischen Veränderungen bei „Leberfibrose“ oder „Nephrosklerose“. Dabei liegen diesen fibrosierenden Veränderungen häufig ganz unterschiedliche Krankheitsursachen zugrunde. Nicht selten sind es entzündliche Veränderungen wie Autoimmunprozesse, die im Lauf der Zeit die chronische Fibrosierung nach sich ziehen.

\section{(D) Der auf den ersten Blick uniforme Prozess der Fibrosierung ist vielgestaltig und wird komplex reguliert}

Während die initialen entzündlichen Prozesse in der Regel viel Aufmerksamkeit erfahren haben und zahlreiche pathophysiologische Mechanismen diskutiert werden, ist es um die molekularen Mechanismen und damit um die zugrunde liegende Pathophysiologie der Fibrose lange Zeit ruhig geblieben. Die fibrosierenden Veränderungen galten als ,unspezifische" vernarbende Endstrecke einer spezifischen Erkrankung. In den letzten Jahren sind jedoch molekulare Mechanismen wie das Transforming-GrowthFactor- (TGF- $\beta$-) System und andere molekulare Mechanismen näher untersucht worden. Damit wird deutlich, dass dieser, auf den ersten Blick uniforme Prozess der Fibrosierung vielgestaltig ist und komplex reguliert wird. Eine Kenntnis der fibrosierenden Mechanismen und der daran beteiligten Zellen und Moleküle liefert uns nicht nur ein besseres Verständnis chronischer Erkrankungen, sondern bietet auch die Möglichkeit, gezielt therapeutisch in den fibrosierenden Prozess einzugreifen und damit den schleichen Verlust der Organfunktion zu verhindern bzw. zu verlangsamen.

Die verschiedenen Beiträge dieses Hefts beleuchten diese Problematik aus unterschiedlichen Richtungen. Prasse, Holle und Müller-Quernheim geben eine Übersicht der Lungenfibrosen. Sie fassen den Stand der Wissenschaft zusammen, geben einen Einblick in die unterschiedlichen Ursachen der Lungenfibrose und erläutern die aktuelle Klassifikation. Sie gehen außerdem auf die (bislang wenigen) therapeutischen Ansätze bei der chronischen Lungenfibrose ein. Wasmuth und Trautwein berichten über die Klinik, Diagnostik und die Therapie der Leberfibrose. Neben der Bedeutung der Leberbiopsie für die Diagnose erläutern sie neuere Verfahren wie Fibroscan und diskutieren die Rolle von Biomarkern. Auch in diesem Beitrag werden, neben der Therapie der jeweiligen Grunderkrankung, neue antifibrotische Therapiestrategien vor dem Hintergrund eines zunehmenden Verständnisses der Leberfibrose erläutert. Diese Thematik steht ganz im Vordergrund der Arbeit von Tacke und Weiskirchen. Hier werden die Rolle von Zytokinen und Chemokinen in der Pathogenese der Leberfibrose beschrieben und neue Therapiestrategien daraus abgeleitet.

Die drei anderen Arbeiten des Hefts beschäftigen sich im Unterschied dazu mit fibrosierenden Erkrankungen, die rasch progredient und durch eine überschieBende Matrixbildung in verschiedenen Organen gekennzeichnet sind. Möglicherweise führt bei diesen Erkrankungen nicht der auslösende Faktor über den Umweg der Entzündung zur Fibrose, sondern induziert Inflammation und Fibrosierung gleichzeitig. Distler und Gay beschreiben den aktuellen Stand der Sklerodermie und arbeiten die pathophysiologischen Mechanismen der Gefäßerkrankung und der raschen Fibrosierung heraus. Die Schwierigkeiten, die Erkrankung zu diagnostizieren, und die klinischen Manifestationen sowie die jetzige Klassifikation der Erkrankung werden erläutert. Kielstein und Schiffer beschreiben die seltene, aber schwerwiegende nephrogene systemische Sklerose. Im Unterschied zu den anderen Erkrankungen ist die Ursache, Gadolinium, bekannt. Risiken in der Verwendung von Kontrastmedien bei Patienten mit Niereninsuffizienz werden thematisiert. „Last but not least" beschreiben Pipitone, Salvarani und Peter die chronische Periaortitis. Auch hier wird deutlich, welche große Rolle die Entzündung der Gefäße für die Pathogenese der fibrosierenden Erkrankungen spielen.

Wir wünschen Ihnen eine interessante Lektüre und viel Vergnügen mit diesem Heft des Internisten.

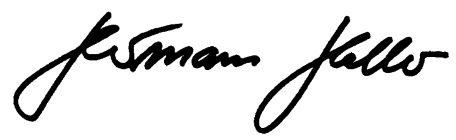

H. Haller

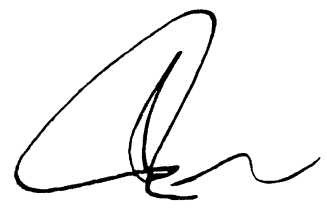

W.L. Gross

\section{Korrespondenzadresse \\ Prof. Dr. H. Haller}

Abteilung Nephrologie,

Medizinische Hochschule Hannover

Carl-Neuberg-Straße 1, 30625 Hannover

haller.hermann@mh-hannover.de 